\title{
Genotypic characterization of Streptococcus pneumoniae serotype 19F in Malaysia
}

\begin{abstract}
Streptococcus pneumoniae is an epidemiologically important bacterial pathogen. Recently, we reported the antibiotic susceptibility patterns of a limited collection of pneumococcal isolates in Malaysia with a high prevalence of erythromycin resistant strains. In the present study, 55 of the pneumococcal isolates of serotype $19 \mathrm{~F}$ were further analysed by pulsed field gel electrophoresis (PFGE) and multilocus sequence typing (MLST). The generated genotypic patterns were then correlated with the antibiograms previously reported. Fortyseven different PFGE profiles (PTs) were obtained, showing that the isolates were genetically diverse. MLST identified 16 sequence types (STs) with ST-236 being predominant $(58.2 \%)$, followed by ST-81 (10.3\%). Among the ST-236 isolates, 22 were erythromycin resistant S. pneumoniae (ERSP) and 15 were trimethoprim/sulfamethoxazole (TMP/SMX) resistant, while among ST-81, four isolates were ERSP and two were TMP/SMX resistant. The high prevalence of erythromycin resistant serotype $19 \mathrm{~F}$ isolates of ST-236 in this study has also been reported in other North and South East Asian countries.
\end{abstract}

Keyword: Streptococcus pneumoniae; Serotype 19F; Antibiogram; Multilocus sequence type; Pulsed field gel electrophoresis 